Artículo científico

(Original paper)

\title{
DIVERSIDAD Y COMPOSICIÓN DE AVES DEL PARQUE NACIONAL LAGUNAS DE MONTEBELLO, CHIAPAS, MÉXICO
}

\section{AVIAN DIVERSITY AND COMPOSITION IN LAGUNAS DE MONTEBELLO NATIONAL PARK, CHIAPAS, MEXICO}

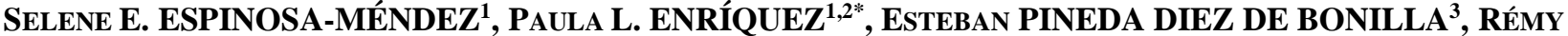 \\ VANDAME ${ }^{4}$ \\ ${ }^{1}$ Departamento Conservación de la Biodiversidad. El Colegio de la Frontera Sur, C.P. 29290, San Cristóbal de Las Casas, \\ Chiapas, México.<selene.esme89@gmail.com>; <penrique@ecosur.mx> \\ ${ }^{2}$ Centro de Investigaciones Biológicas del Noroeste. Av. Instituto Politécnico Nacional 195, Col. Playa Palo de Santa Rita Sur, \\ C.P. 23096, La Paz, Baja California Sur, México. <penrique@ecosur.mx> \\ ${ }^{3}$ Instituto de Ciencias Biológicas. Universidad de Ciencias y Artes de Chiapas, C.P. 29000, Tuxtla Gutiérrez, Chiapas, México. \\ <esteban.pineda@unicach.mx> \\ ${ }^{4}$ Departamento Agricultura, Sociedad y Ambiente. El Colegio de la Frontera Sur, C.P. 29290, San Cristóbal de Las Casas, \\ Chiapas, México.<remy@ecosur.mx> \\ *Autor corresponsal: <penrique@ecosur.mx>
}

Recibido: 26/07/2018; aceptado: 20/04/2020; publicado en línea: 10/06/2020

Editor responsable: Octavio Rojas Soto

Espinosa-Méndez, S. E., Enríquez, P. L., Pineda-Diez de Bonilla, E., Vandame, R. (2020) Diversidad y composición de aves del Parque Nacional Lagunas de Montebello, Chiapas, México. Acta Zoológica Mexicana (nueva serie), 36, 1-16. https://doi.org/10.21829/azm.2020.3612062

RESUMEN. Actualmente los ambientes heterogéneos son los que predominan en los paisajes tropicales, por lo que estudiar la diversidad y la composición de especies en las comunidades avifaunísticas ha permitido entender la variación y la estructura de las mismas. Asimismo, esta información permite implementar estrategias de manejo y conservación en estos ambientes. En este estudio se evaluó la diversidad y composición espacial y temporal de la comunidad de aves en el Parque Nacional Lagunas de Montebello, Chiapas, en diferentes tipos de bosques y condiciones (bosques de pino, pino-encino, pinoencino-liquidambar, bosque mesófilo, vegetación riparia y zonas intervenidas). El muestreo se realizó de febrero a julio de 2017 en 110 puntos de conteo separados cada $300 \mathrm{~m}$. En total se registraron 3,143 aves de 123 especies. La diversidad medida con el orden $q=1$ fue de 41.67 y con $q=2$ fue de 24.79 especies efectivas. La temporada de lluvias presentó una mayor diversidad de especies de aves que en secas. Los gremios mejor representados fueron los granívoros-frugívoros-insectívoros, los insectívoros y los omnívoros. Las zonas con intervención, así como los bosques de pino y la vegetación riparia presentaron la mayor diversidad de especies. En contraste, el bosque mesófilo de montaña, de pino-encino-liquidambar y de pino-encino presentaron especies raras y vulnerables como Setophaga chrysoparia, Pharomachrus mocinno y Penelopina nigra. Este estudio permite identificar sitios de monitoreo y de manejo forestal para el mejor ordenamiento en el parque nacional.

Palabras clave: abundancia; bosques de montaña; conservación; gremios; manejo; riqueza de especies 
Espinosa-Méndez, S. E., Enríquez, P. L., Pineda-Diez de Bonilla, E., Vandame, R. (2020) Avian diversity and composition in Lagunas de Montebello National Park, Chiapas, Mexico. Acta Zoológica Mexicana (nueva serie), 36, 1-16. https://doi.org/10.21829/azm.2020.3612062

\begin{abstract}
Heterogeneous environments predominate in tropical landscapes, then studying diversity and composition of species in avian communities has allowed understand how it vary and is structured. This information also allows implementing management and conservation strategies in these environments. This study evaluated the diversity and spatial and temporal composition of the bird community in Lagunas de Montebello National Park, Chiapas, in different types of forests and conditions (pine forests, pine-oak, pineoak-liquidambar, mountain cloud forest, riparian vegetation and modified areas). Sampling was carried out from February to July 2017 at 110 counting points every $300 \mathrm{~m}$. In total, 3,143 birds of 123 species were recorded. The diversity measured with the order $\mathrm{q}=1$ was 41.67 and with $\mathrm{q}=2$ it was 24.79 effective species. Rainy season showed greater species diversity than dry season. The best represented guilds were granivoresfrugivores-insectivores, insectivores and omnivores. The modified areas, as well as pine forest and riparian vegetation areas had the greatest diversity of species. In contrast, mountain cloud forest, pine-oakliquidambar, and pine-oak forests had rare and vulnerable species such as Golden-cheeked Warbler, Resplendent Quetzal, and Highland Guan. This study allows to identify sites for monitoring and forest management that will allow for better planning in the national park.
\end{abstract}

Key words: abundance; mountain forests; conservation; guilds; management; species richness

\title{
INTRODUCCIÓN
}

Entender cómo se estructuran las comunidades ecológicas y como varía su diversidad y composición en el tiempo y espacio en paisajes heterogéneos permitirá identificar sitios importantes para la conservación y restauración de bosques. Uno de los ecosistemas que son prioritarios para la conservación por su diversidad y presencia de especies endémicas, pero también por su importancia en los ciclos hidrológicos y de nutrientes, son los bosques de montaña (CONABIO, 2010). Los bosques tropicales de montaña en Mesoamérica, son el segundo más importante hot spot de biodiversidad para el mundo (Myers et al., 2000), son reconocidos por su alto endemismo (Renjifo et al., 1997), pero son uno de los ecosistemas más amenazados (Kappelle \& Brown, 2001). Esta amenaza es debido al cambio en el uso del suelo para áreas de uso agrícola, pastoreo, tala para leña y uso forestal y crecimiento urbano (Stattersfield et al., 1998), pero también por cambios naturales como tormentas tropicales, incendios y plagas forestales.

Las aves desempeñan un importante papel en la dinámica y funcionalidad de los ecosistemas (Şekercioḡlu, 2006; Sohdi et al., 2011), y estudiarlas ha permitido entender procesos ecológicos y funcionales (Şekercioğlu, 2006). En estos bosques de montaña se concentra una alta diversidad de especies de aves, en México, el $47 \%$ de las especies en el país se distribuyen en estos bosques (Navarro-Singüenza et al., 2014). Las aves son un grupo taxonómico que ha sido modelo para identificar áreas prioritarias de conservación e implementar estrategias de conservación y manejo (Navarro-Sigüenza et al., 2011).

La pérdida de cobertura forestal en Chiapas se estimó que para un lapso de casi 20 años (19902009) fue de 2,202 km², y la degradación forestal (cobertura menor al 30\%) ascendió a 7,693 km² (Paz et al., 2010). Particularmente, en el Parque Nacional Lagunas de Montebello (PNLM, en adelante) Chiapas, que es reconocido por la belleza escénica de sus lagos, aún resguarda remanentes característicos de bosques de montaña. Con el decreto del parque en 1959, se creó una frontera de conservación en donde las localidades aledañas se vieron limitadas en el aprovechamiento de sus recursos naturales. Pero durante el periodo del 2005 al 2009, la poligonal del parque (6,425 ha) presentó una transformación de su masa forestal 2 
de 43 hectáreas (33.9\%), por el aumento del cultivo de maíz y café (Jiménez-Cruz \& Pulido-Argüello, 2009). A pesar de estos cambios en el área, poca información se ha generado sobre los cambios y variaciones en las comunidades avifaunísticas. Rangel-Salazar et al. (2009), estudiaron la comunidad de aves en el PNLM en diferentes condiciones de perturbación y encontraron que el sitio más perturbado no presentó la mayor riqueza, pero si la menor diversidad de aves. Nuestra predicción fue que la riqueza, diversidad y composición de especies de aves presentarán una variación de acuerdo con el tipo de vegetación, y también habrá una variación temporal. Este tipo de análisis son una herramienta útil que además de tener un enfoque de conservación, evalúa los cambios en los ambientes naturales y perturbados (Toledo, 1994). En este estudio el objetivo fue evaluar la variación espacial y temporal de la diversidad y composición de aves en diferentes tipos de vegetación en el Parque Nacional Lagunas de Montebello, Chiapas, México. Esto permitirá la formulación de estrategias de manejo con los pobladores de las comunidades aledañas al parque, para continuar con las actividades productivas de la región pero que mitiguen la transformación de estos ecosistemas.

\section{MATERIALES Y MÉTODOS}

Zona de estudio. El Parque Nacional Lagunas de Montebello está localizado en la región sur-sureste del estado de Chiapas, en la frontera con Guatemala. Sus coordenadas extremas son $16^{\circ} 09^{\prime} 31^{\prime \prime}$ y $16^{\circ} 04^{\prime} 48^{\prime}$ " Latitud Norte y $91^{\circ} 47^{\prime} 39^{\prime \prime}$ y $91^{\circ} 38^{\prime} 39^{\prime \prime}$ Longitud Oeste (Fig. 1). Presenta una extensión territorial de 6,425 ha en su poligonal y una zona de influencia con una superficie de 8,530 ha que incorpora 13 comunidades humanas mexicanas y guatemaltecas (CONANP, 2007). En el Parque Nacional Lagunas de Montebello se encuentran bosques de pino, pino-encino y pino-encino-liquidambar, como tipos de vegetación dominantes. En menor proporción se encuentra el bosque mesófilo de montaña. También existen numerosos cuerpos de agua, áreas inundables de popal-tular y en la periferia, áreas agrícolas, huertos familiares, cafetales, pastizales inducidos por la acción del ganado o fuego y asentamientos humanos (CONANP, 2007).

Diseño del muestreo. Se recorrieron senderos o caminos rurales para establecer los puntos de muestreo e identificar los tipos de vegetación. Las características que se consideraron fueron sitios accesibles, de recorrido seguro, y tener los permisos con las autoridades institucionales del parque y ejidales correspondientes. Inicialmente se establecieron 116 unidades de muestreo separadas entre sí por $300 \mathrm{~m}$, las cuales estuvieron distribuidas en 10 senderos de longitud variable. La distancia entre unidades (puntos de conteo) es la distancia sugerida para permitir independencia en los registros (Hutto et al., 1986; Ralph et al., 1996). Durante el muestreo, seis puntos tuvieron que ser eliminados por razones de seguridad. Las unidades de muestreo se agruparon de acuerdo con las características de la vegetación: 28 unidades en bosque de pino (BP); 17 en bosque de pino-encino (BPE); 32 en bosque de pino-encino-liquidambar (BPEL); 5 en vegetación riparia (VR); 9 en bosque mesófilo de montaña (BMM) y 19 en zonas con intervención (ZI; Fig. 1). La vegetación dominante en el área son los bosques de pino, pino-encino y pinoencino-liquidambar, y a continuación se describe cada uno de estos bosques. Las zonas con intervención se definieron como unidades que no presentan una comunidad florística definida y que son consecuencia de factores naturales o antropogénicos (i.e. cultivos de maíz, cafetales, potreros, cultivos abandonados o sitios afectados por incendios forestales). Aunque en la zona intervenida estos ambientes pueden ser contrastantes, son ambientes que fueron transformados casi completamente de su vegetación original, por esta razón se consideraron como zonas intervenidas. Hay especies de aves características de bosques que no se encontraron en estos ambientes intervenidos.

El bosque de pino presenta una amplia distribución en el PNLM, abarcando toda la porción centrooeste. Es florísticamente pobre, la mayoría de estas especies están adaptadas a condiciones extremas de 
temperatura y radiación solar. Este bosque está asociado a sitios con menor pendiente y elevación dentro del parque, además, es donde la afectación por incendios forestales superficiales ha sido frecuente (Melo \& Cervantes, 1986; CONANP, 2007). El bosque de pino-encino presenta especies de Pinus y Quercus que dominan el dosel. La co-dominancia del encino permite una gran riqueza y abundancia de epífitas, como bromelias, orquídeas y helechos (González-Espinosa \& Ramírez-Marcial, 2013). El bosque de pino-encinoliquidambar se distribuye en la parte este del parque, su característica distintiva es la presencia de Liquidambar styraciflua en codominancia con Quercus spp. y Pinus spp. Este bosque presenta una alta riqueza de especies florísticas y, además, resguarda un número importante de especies vegetales asociadas a sitios pocos perturbados. El bosque mesófilo de montaña presenta una distribución reducida en comparación con las otras asociaciones forestales. En el 2005 se reportó una superficie de 458 ha (7.13\%) y está restringido a áreas con características de topografía abrupta y laderas, específicamente en la parte noreste y central del parque (Jiménez-Cruz \& Pulido-Arguello, 2009). Este tipo de bosque presenta una gran riqueza de especies leñosas, frecuentemente numerosas lauráceas y especies importantes que se encuentran bajo alguna categoría de riesgo (CONANP, 2007). La vegetación riparia está asociada a los lechos poco profundos de la orilla de algunos lagos y se reconoce principalmente por la presencia de tulares (Typha sp.) y carrizales (Pragmites sp.; CONANP, 2007). Es importante mencionar, que el criterio utilizado para agrupar estas unidades de muestreo $(\mathrm{N}=5)$ fue principalmente por poseer $>50 \%$ de los elementos florísticos que caracterizan a este tipo de vegetación.

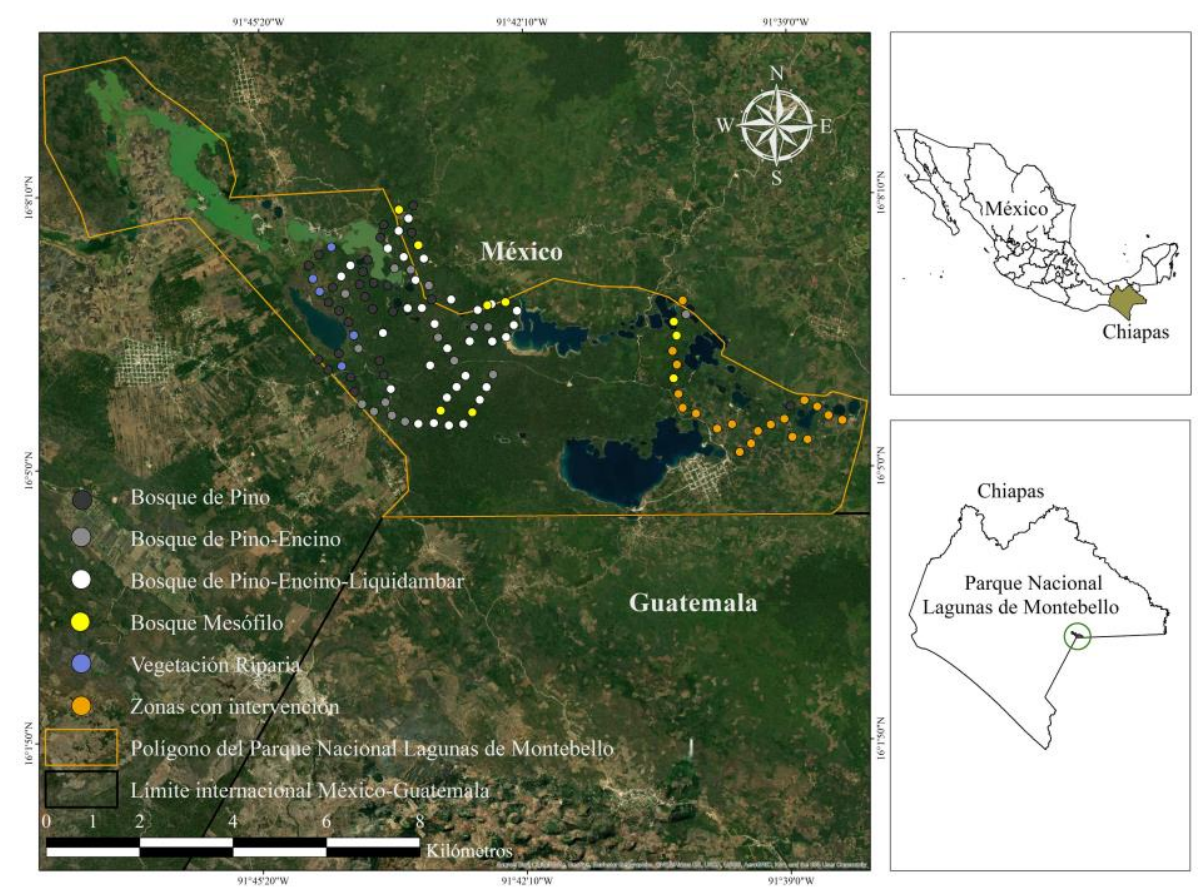

Figura 1. Localización geográfica del Parque Nacional Lagunas de Montebello, Chiapas y de los puntos de conteo por tipo de vegetación.

En cada punto seleccionado, se realizó el conteo de aves (vistas o escuchadas) con un radio fijo de $25 \mathrm{~m}$ durante $10 \mathrm{~min}$ (Bibby, 2000). Los muestreos se llevaron a cabo de 6:00 am a 11:00 am. Cada unidad de muestreo fue visitada una vez al mes por seis meses, tres en la temporada de secas (febrero- abril de 2017) y tres en lluvias (mayo-julio de 2017). Para disminuir los sesgos de observación, los recorridos iniciaron a diferentes horarios y de forma aleatoria, y la separación temporal entre visitas en un mismo punto fue de 30 días. 
Para los análisis de la composición de la comunidad de aves, las especies se agruparon en gremios tróficos: granívoros-frugívoros-insectívoros (GFI), insectívoros (I), omnívoros (O), insectívorosvertebrados pequeños (IVp), insectívoros-nectarívoros (IN), granívoros (G), frugívoros-insectívorosvertebrados pequeños (FIVp), insectívoros-invertebrados acuáticos-vertebrados pequeños (IAcVp) e insectívoros-frugívoros (IF), con base en Rangel-Salazar et al. (2009), Ruiz-Bruce-Taylor et al. (2017) y The Cornell Lab of Ornithology (2017). Con respecto al estrato de forrajeo, las especies fueron clasificadas en: agua ( $\mathrm{Ag})$, terrestre (Te), sotobosque (So), estrato medio (EM), estrato alto (EA) y aéreo (V); por su sensibilidad se siguió la propuesta de Stotz et al. (1996) como especies con baja (B) sensibilidad a la perturbación, con media (M) sensibilidad y alta (A) sensibilidad, y por su estatus migratorio en residentes y migratorias latitudinales. El listado taxonómico fue con base al listado del American Ornithologist's Union (Chesser et al., 2019).

Análisis de Datos. La diversidad 9D (sensu Jost, 2006) se midió como números efectivos de Hill (1973) en tres órdenes $q=0,1$ y 2 . Se realizó este análisis, porque transformar los índices o entropías a números efectivos de especies es importante para una mejor interpretación unificada de la diversidad, ya que se obtiene una medida de similitud general, estable y es de fácil interpretación (Jost, 2006; Chao, 2014). De esta forma ${ }^{0} \mathrm{D}$ corresponde a la riqueza total de especies en la muestra, ${ }^{1} \mathrm{D}$ mide la diversidad considerando todas las especies con un peso exactamente proporcional a su abundancia, además que es una medida equivalente al exponencial del índice de Shannon, y ${ }^{2} \mathrm{D}$ mide la diversidad de las especies más dominantes y es una medida equivalente al inverso del índice de Simpson (Jost, 2006). Estos tres valores de diversidad se calcularon de manera general para todo el inventario por temporada y tipo devegetación. La cobertura de la muestra y las medidas de diversidad se estimaron con $\mathrm{R}$ con el paquete iNEXT (Hsieh et al., 2016) Se realizaron análisis de varianza (ANOVA) y Kruskal-Wallis, para comparar las variables según fuera el caso mediante el programa InfoStat versión 2008.

\section{RESULTADOS}

En total se registraron 3,143 individuos de aves durante seis meses, pertenecen a 13 órdenes, 31 familias y 123 especies. La Familia Parulidae fue la mejor representada en riqueza y abundancia (17 especies y 398 individuos), seguida de Turdidae (11 especies y 630 individuos). En contraste, Alcedinidae tuvo un individuo de la especie Megaceryle torquata. Las especies abundantes fueron Myadestes occidentalis (260 individuos), Myioborus miniatus (258 individuos), Icterus chrysater (245 individuos), Ortalis vetula (212 individuos) y Chlorospingus flavopectus (168 individuos). El $62.9 \%$ de la riqueza total de aves fueron especies poco comunes, con abundancias $\leq 10$ individuos, y la cobertura de la muestra estimada con el esfuerzo de muestreo invertido para el parque fue del 99\% (Apéndice 1).

La diversidad ${ }^{1} \mathrm{D}$ y ${ }^{2} \mathrm{D}$ fue de 41.67 y 24.79 especies efectivas, respectivamente (Cuadro 1 ). Al realizar las comparaciones de estas estimaciones entre ambas temporadas, todas fueron diferentes: ${ }^{0} \mathrm{D}: \mathrm{F}_{5,6}=$ 14.3, $\mathrm{P}=0.01,{ }^{1} \mathrm{D}: \mathrm{F}_{5,6}=29.9, \mathrm{P}=0.005$ y ${ }^{2} \mathrm{D}: \mathrm{F}_{5,6}=20.5, \mathrm{P}=0.01$; en estos tres parámetros, los valores fueron mayores en la temporada de lluvias. Las estimaciones de diversidad fueron diferentes entre los tipos de vegetación $\left({ }^{0} \mathrm{D}: \mathrm{F}_{5,18}=23.9, \mathrm{P}=\left\langle 0.0001 ;{ }^{1} \mathrm{D}: \mathrm{H}_{5,18}=12.3, \mathrm{P}=0.03,{ }^{2} \mathrm{D}: \mathrm{H}_{5,18}=13.9, \mathrm{P}=0.01\right)\right.$. Las zonas con intervención (ZI) presentaron la mayor diversidad total ${ }^{1} \mathrm{D}=48.35$ y ${ }^{2} \mathrm{D}=32.33$ ) con respecto a toda el área muestreada (Cuadro 1). En contraste, el bosque de pino-encino (BPE) presentó los valores más bajos de diversidad ${ }^{1} \mathrm{D}$ y ${ }^{2} \mathrm{D}$ (Cuadro 1).

Con respecto a la composición de especies de aves en la comunidad, la mayoría fueron especies residentes y un 6.8\% (20) migratorias latitudinales. Los gremios mejor representados fueron los granívorosfrugívoros-insectívoros (GFI) con 53 especies y los insectívoros con 24 especies (Apéndice 1). Por otra 
parte, los insectívoros-frugívoros (IF) y los frugívoros-insectívoros-vertebrados pequeños (FIVp) fueron los menos representados con dos y cuatro especies, respectivamente (Fig. 2). El estrato alto (Ea) fue el más utilizado por la comunidad de aves para forrajear, seguido del estrato medio-alto (Em, Ea) y el sotobosqueestrato medio (So, Em). La mayor riqueza y abundancia de aves se registró durante la temporada de lluvias (mayo-julio) con 1,950 individuos de 107 especies, respecto a la temporada de secas (febrero-abril) con 1,194 individuos de 87 especies.

Cuadro 1. Diversidad de especies de aves por temporada y tipo de vegetación en el Parque Nacional Lagunas de Montebello, Chiapas, México.

\begin{tabular}{ccccc}
\hline \multirow{2}{*}{ PNLM } & & \multicolumn{3}{c}{ Números efectivos de especies } \\
\cline { 3 - 5 } & & $\mathbf{0} \mathbf{D}$ & $\mathbf{1}$ D & 'D \\
\cline { 3 - 5 } & & 123 & 41.67 & 24.79 \\
\hline \multirow{2}{*}{ Temporada } & Lluvias & 107 & 41.4 & 25.53 \\
& Secas & 87 & 31.52 & 19.43 \\
\hline \multirow{3}{*}{ Tipo de vegetación } & ZI & 87 & 48.35 & 32.33 \\
& BP & 72 & 32.7 & 22.3 \\
& BPEL & 66 & 26.03 & 16.58 \\
& BPE & 54 & 24.09 & 16.29 \\
& BMM & 49 & 27.31 & 18.54 \\
& VR & 40 & 27.73 & 21.75 \\
\hline
\end{tabular}

Número efectivo de especies: ${ }^{0} \mathrm{D}$ : riqueza; ${ }^{1} \mathrm{D}$ : diversidad de orden $1 ;{ }^{2} \mathrm{D}$ : diversidad de orden 2 . Tipos de vegetación: $\mathrm{ZI}=$ Zonas con intervención; $\mathrm{BP}=\mathrm{Bosque}$ de pino; $\mathrm{BPEL}=\mathrm{Bosque}$ de pino-encino-liquidambar; $\mathrm{BPE}=\mathrm{Bosque}$ de pinoencino; $\mathrm{BMM}=$ Bosque mésofilo de montaña; $\mathrm{VR}=$ Vegetación riparia.

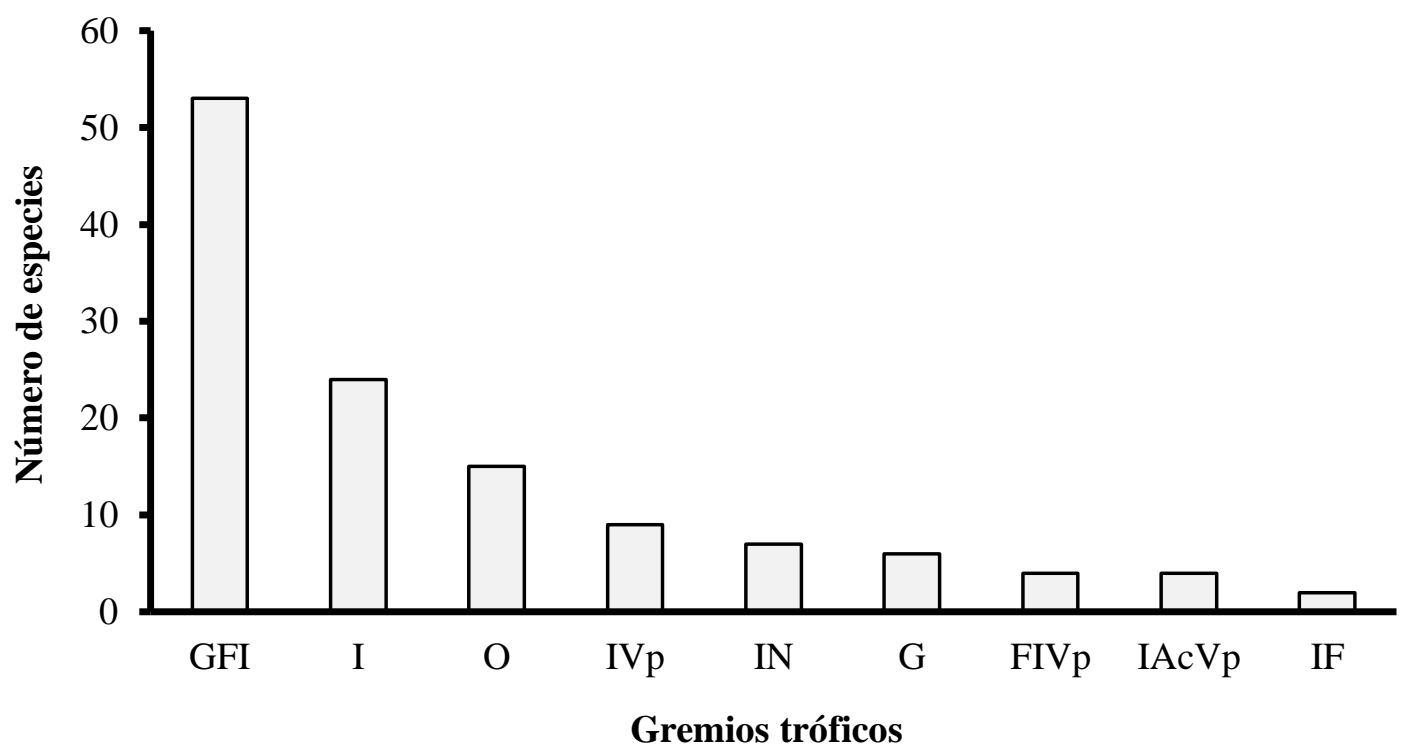

Figura 2. Gremios tróficos en seis tipos de vegetación de febrero-julio de 2017 en el Parque Nacional Lagunas de Montebello, Chiapas. Gremios: GFI=granívoro-frugívoro-insectívoro; I=Insectívoro; O=Omnívoro;

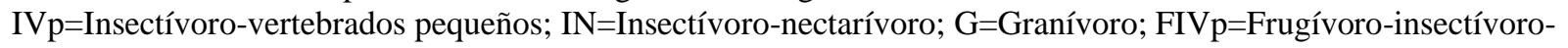
vertebrados pequeños; IAcVp= Insectívoro-invertebrados acuáticos-vertebrados pequeños; IF= Insectívoro-

frugívoro. 
Las zonas con intervención (ZI) presentaron la mayor riqueza de especies (87 especies). Veinticuatro especies se registraron exclusivamente en estas zonas y la mayoría de ellas presentan de baja a mediana sensibilidad a la perturbación de acuerdo con la clasificación de Stotz et al. (1996) (Apéndice 1). El bosque de pino (BP) presentó 72 especies y 844 individuos, y el bosque de pino-encino-liquidambar (BPEL) 66 especies y 790 individuos (Cuadro 1). Las aves presentes en el BPEL fueron, en su mayoría, especies de mediana a alta sensibilidad a la perturbación. Generalmente, las especies emplearon el estrato alto para forrajear, aunque en el BMM y la VR la mayoría de las especies forrajearon en el estrato medioalto.

\section{DISCUSIÓN}

Las estimaciones de diversidad y composición espacial y temporal en diferentes tipos de vegetación permiten entender cómo se estructuran las comunidades y cómo éstas varían en el tiempo. En este estudio se registraron en total 123 especies de aves, que representaron el 17.5\% del total de aves reportadas para el estado de Chiapas (Rangel-Salazar et al., 2013) y el 44.4\% de las especies de aves registradas en el PNLM (CONANP, 2007). El esfuerzo de muestreo realizado en este estudio presentó una buena cobertura de la muestra. Se ha reportado que, en bosques, el utilizar los registros auditivos de aves permite detectar aquellas especies raras, poco conspicuas o las que son muy activas en el dosel y dificultan su registro visual (CelisMurillo et al., 2012; Suárez-García et al., 2017). Por lo tanto, es importante utilizar ambos métodos (visual y auditivo) para tener una mejor aproximación de los resultados.

Las Familias Parulidae y Turdidae han sido reportadas como representativas del parque (MartínezSánchez, 2005; Rangel-Salazar et al., 2009) así como en algunos bosques de Chiapas, Guerrero y el Estado de México (Bojoges-Baños, 2004; Almazán-Núñez et al., 2009; Rangel-Salazar et al., 2013). En contraste, una de las familias menos representada fue Alcedinidae, con un solo individuo de Megaceryle torquata. En la zona donde se registró esta especie hay cierto grado de perturbación con constante afluencia de paso vehicular, de pescadores y un lago impactado con una disminución de peces (Maya-García, 2017). De acuerdo con Durán (2017), las poblaciones de M. torquata pueden verse afectadas por la disminución de sus recursos dulceacuícolas y pérdida de sus sitios de nidificación.

Las especies que presentaron mayores abundancias (I. chrysater, O. vetula, M. occidentalis, $M$. miniatus, y $C$. flavopectus) se registraron en ambas temporadas, pero también presentaron una amplia distribución con registros en todos los tipos de vegetación. Las especies Myadestes occidentalis, Mioborus miniatus y Chlorospingus flavopectus son consideradas comunes en las Lagunas de Montebello, pero también en otros bosques templados del país (Martínez-Sánchez, 2005; Navarro \& Peterson, 2007; RangelSalazar et al., 2009; Almazán-Núñez et al., 2009; Rangel-Salazar \& Enríquez, 2013). Asimismo, en estos bosques de montaña también son características las especies raras o endémicas, las cuales son prioritarias para la conservación. En este estudio se registraron Setophaga chrysoparia (1), Cyanolyca pumilo (2), Pharomachrus mocinno (2), Cyanolyca cucullata (4), Aspatha gularis (7) y Catharus frantzii (8), ya que además de ser poco comunes, son consideradas especies amenazadas o en peligro de extinción en México (NOM-059 SEMARNAT-2010; Apéndice 1). La comunidad de aves estuvo integrada en su mayoría por especies de baja a mediana sensibilidad a la perturbación de acuerdo con la clasificación de Stotz et al. (1996). Sobresalen Penelopina nigra, Turdus infuscatus, Dendrocolaptes picumnus, A. gularis у C. pumilo por ser especies con alta sensibilidad a la perturbación (Apéndice 1).

La zona intervenida (ZI) presentó la mayor riqueza de especies (87 especies). De todos los sitios estudiados, esta zona tuvo la mayor heterogeneidad por los diferentes usos de suelo presentes en el área, lo que permiten una mayor diversidad y riqueza de especies. En la vegetación riparia (VR) se registraron 
especies que utilizan hábitat abiertos como Zenaida asiática y Amazilia cyanocephala (Van Perlo, 2006). Por otro lado, I. chrysater y $O$. vetula no se han reportado abundantes en bosques, pero también en vegetación secundaria (CONANP, 2013). Posiblemente, los hábitos generalistas de estas especies, su baja sensibilidad a la perturbación, su gran movilidad en ambientes perturbados y la ubicación del parque como corredor biológico con la Selva Lacandona, sean características que están favoreciendo la ocurrencia de estas especies en el parque.

La diversidad de especies efectivas dominantes $\left({ }^{2} \mathrm{D}\right)$ reportadas en este estudio fue 1.8 veces mayor (55.14\%) que la diversidad reportada anteriormente (Rangel-Salazar et al., 2009), posiblemente por los métodos de muestreo utilizados (redes de niebla utilizadas en el estudio previo), y características espaciotemporales de los muestreos. Las redes de niebla están sesgadas a las aves de sotobosque y cubren menor área por unidad de tiempo; a diferencia de los puntos de conteo, que además de cubrir una mayor área, permiten detectar especies raras, poco conspicuas y de dosel (Ralph et al., 1996). Esta diferencia en la diversidad de aves de Montebello indica que la estructura de la comunidad puede ser una asociación peculiar y altamente dinámica (con pérdida y ganancia de especies), aun cuando la estructura básica permanezca (Halffter \& Moreno, 2005). Asimismo, la variabilidad encontrada refleja la necesidad de realizar estudios a largo plazo, que permitan entender la dinámica de la diversidad en la comunidad avifaunística como respuesta a factores naturales (e.g. incendios forestales) o antropogénicos (e.g. cambio de uso de suelo) que inciden en el área protegida (Jiménez-Cruz \& Pulido-Arguello, 2009; Sohdi et al., 2011).

La temporada de lluvias presentó un aumento en la diversidad ${ }^{1} \mathrm{D}$ y ${ }^{2} \mathrm{D}$ que se explica por el incremento en la abundancia de las especies comunes, principalmente las especies insectívoras (I), granívoras-frugívoras-insectivoras (GFI) e insectívoras-nectarívoras (IN). La abundancia de estos gremios responde positivamente al incremento en los niveles de recursos (néctar, insectos, frutos) durante esta temporada, los que a su vez se asocian por la disponibilidad estacional de los recursos en los sitios (e.g. presencia de flores para insectos polinizadores; madera en descomposición para coleópteros; Codesido \& Bilenca, 2004). De igual forma, las lluvias favorecen la abundancia de insectos con ciclos de vida cortos (e.g. Melolonthidae), lo que se traduce en una mayor oferta de recursos para las aves. Estudios a largo plazo permitirán comparar las fluctuaciones estacionales de los recursos y su efecto en la estructura de la comunidad avifaunística del parque.

La diversidad de aves en los bosques que presentan una mayor riqueza de especies florísticas y sitios menos perturbados como el BMM, BPEL y BPE fueron menores con relación a los tipos de vegetaciones de zonas abiertas (ZI, VR y BP). Es posible que la densa cobertura del dosel en estos bosques, la experiencia del observador y el tipo de muestreo hayan influido en el menor registro de especies y abundancia de aves y, por ende, menor diversidad. Se ha reportado que, en follajes densos o muy altos, la detección de las aves se dificulta existiendo omisiones en los registros (González-Ortega \& Morales-Pérez, 1998). Sin embargo, sobresale la composición de especies en estos sitios boscosos, por ser aves residentes con mediana y alta sensibilidad a la perturbación, principalmente, Atlapetes albinucha, Penelopina nigra y Pharomachrus mocinno (Stotz et al., 1996). Además, son especies de bosque (carpinteros, trepatroncos, trogones, zorzales, mirlos, y tángaras) que dependen de los árboles para sobrevivir (Lammertink et al., 1996).

En contraste, la diversidad de aves en la vegetación riparia, zona con intervención y bosque de pino estuvo compuesta principalmente por especies dominantes con baja sensibilidad a la perturbación. En cada tipo de vegetación, se registraron especies exclusivas asociadas a hábitats abiertos, así como algunas especies de interior de bosque (e.g. Turdus infuscatus). Nuestros resultados sugieren que estos tipos de vegetación representan una opción para las aves residentes que pueden desplazarse entre ambientes en búsqueda de recursos (Şekercioḡlu et al., 2002). 
En las ZI, Cyanerpes cyaneus, Volatinia jacarina y Sporophila torqueola fueron especies exclusivas en estos sitios. Para Dendrocygna autumnalis, la VR fue adecuada para anidar por la presencia de pastizales densos y su cercanía a los cuerpos de agua. Debido a la constante trasformación de los hábitats originales, es importante mantener sitios naturales y modificados para conservar la estructura y complementariedad de una comunidad (Ramírez-Albores, 2006). También, sobresale la presencia de Bubulcus ibis en las zonas con intervención, al ser una especie exótica genera competencia por sitios de anidación y alimento con las aves residentes y transportan la bacteria Clostridium botulinum que puede afectar a otras aves y mamíferos (Sánchez-García, 2012).

Por otra parte, es probable que la diversidad en el BP esté moldeada por la intensa actividad de reforestación y el manejo forestal para la erradicación de la plaga de descortezador, que involucra principalmente el derribo de árboles infestados. Esta apertura del dosel podría beneficiar aquellas especies que utilizan hábitats abiertos, como Zenaida asiática, Quiscalus mexicanus, O. vetula e I. chrysater, que fueron abundantes en estos sitios. La modificación en el dosel (mayores claros), la heterogeneidad y arquitectura de la vegetación, influyen en la diversidad, surgiendo mecanismos que favorecen la coexistencia de las especies. Asimismo, la familia Picidae fue representativa en términos de riqueza de especies y abundancia de individuos en el BP; por lo que podrían ser indicadoras del disturbio causado por el manejo forestal en estos sitios. De acuerdo con Sobrino-Galdámez (2011), estas aves son anidadoras primarias de cavidades, y pueden verse afectadas de manera directa y negativa por un manejo constante del bosque y afectar de forma indirecta a otras especies participantes en las redes de anidación en bosques templados.

En este estudio, los gremios GFI, I y $\mathrm{O}$ fueron representativos en términos de riqueza, como se reportó previamente por Rangel-Salazar et al. (2009). Los GFI e I son de vital importancia para la dispersión de semillas y el control de plagas en el parque, además, están relacionados a hábitats con mayor complejidad estructural y florística (Ramírez-Albores, 2006). Las especies insectívoras son sensitivas y vulnerables a la fragmentación, y su persistencia depende de la capacidad para moverse entre ambientes perturbados. Si estas especies son poco móviles y exclusivas de interior de bosque, están más amenazadas a desaparecer (Şekercioḡlu et al., 2002). En este trabajo, las especies insectívoras fueron más abundantes en el BPEL (bosque maduro), pero también en ZI (zonas con intervención). Respecto al alto número de especies omnívoras $(\mathrm{O})$ en el parque, puede deberse a un reflejo de la heterogeneidad de la vegetación, ya que son especies que requieren hábitats menos específicos y un uso más amplio del espacio (Ramírez-Albores, 2006; Pineda-Diez de Bonilla et al., 2012). Estos resultados indican que esta variación en la composición de las comunidades avifaunísticas puede resultar en ocasiones en comunidades menos especializadas y que pueden reducir la función en estos ecosistemas y afectar los servicios ecosistémicos (Şekercioḡlu et al., 2012).

Este estudio permitió la inclusión de cuatro especies al listado avifaunístico del parque (e.g. Dryocopus lineatus, Xiphorhynchus flavigaster, Sayornis nigricans y Setophaga pensylvanica) y nuevas áreas de avistamiento del quetzal. Por la composición de la diversidad avifaunística en el BMM, BPEL y BPE, se enfatiza la necesidad de implementar estrategias de restauración del bosque, formular alternativas de aprovechamiento forestal con los pobladores de las comunidades aledañas al parque y estrategias encaminadas a la ordenación de los espacios de uso público. Para la VR y ZI, es necesario establecer un manejo que evite su transformación en áreas agropecuarias más intensivas. Debido al contexto histórico y social del parque Nacional Lagunas de Montebello, es indispensable considerar la participación de los actores sociales para combinar conocimiento técnico y tradicional y formular alternativas de conservación y manejo en el parque. 
AgradeCIMientos. Al CONACYT, por la beca No. 430477 brindada al primer autor. A ECOSUR por los fondos fiscales para el trabajo de campo. A J. R. Vázquez por su apoyo en el trabajo de campo, identificación de especies y métodos estadísticos. A las brigadas comunitarias, pobladores y manejadores del Parque Nacional Lagunas de Montebello por facilitar la logística durante la fase de campo. Agradecemos al Editor Asociado y revisores por sus acertados comentarios que ayudaron a mejorar sustancialmente el manuscrito.

\section{LITERATURA CITADA}

Almazán-Núñez, R. C., Puebla-Olivares, F., Almazán-Juárez, A. (2009) Diversidad de aves en bosques de pino-encino del centro de Guerrero, México. Acta Zoológica Mexicana (nueva serie), 25, 123 142. Disponible en: http://www.scielo.org.mx/pdf/azm/v25n1/v25n1a11.pdf

Bibby, C., Burgess, N., Hill, D., Mustoe, S. (2000) Bird Census Techniques. 2nd Edition. Academic Press, $302 \mathrm{pp}$.

Bojorges-Baños, J. C. (2004) Riqueza de aves de la región noreste de la Sierra Nevada, Estado de México. Acta Zoológica Mexicana (nueva serie), 20, 15-29.

Celis-Murillo, A., Deppe, J. L., Ward, M. P. (2012) Effectiveness and utility of acoustic recordings for surveying tropical birds. Journal of Field Ornithology, 83, 166-179. https://doi.org/10.1111/j.1557-9263.2012.00366.x

Chao, A., Gotelli, N., Hsieh, T., Sander, E., Colwell, R., Ellison, A. (2014) Rarefaction and extrapolation with Hill numbers: a framework for sampling and estimation in species diversity studies. Ecological Monographs, 84, 45-67. Disponible en: http://www.jstor.org/stable/43187596

Chesser, R. T., Burns, K. J., Cicero, C., Dunn, J. L., Kratter, A. W., Lovette, I. J., Rasmussen, P. C., Remsen, Jr. J. V., Stotz, D. F., Winker, K. (2019) Check-list of North American Birds (online). American Ornithological Society. Disponible en: http://checklist.aou.org/taxa

Codesido, M., Bilenca, D. (2004) Variación estacional de un ensamble de aves en un bosque subtropical semiárido del Chaco Argentino. Biotropica, 36, 544-554. https://doi.org/10.1111/j.1744-7429.2004.tb00349.x

CONABIO (Comisión Nacional para el Conocimiento y Uso de la Biodiversidad) (2010) El bosque mesófilo de montaña en México: amenazas y oportunidades para su conservación y manejo sostenible. Comisión Nacional para el Conocimiento y Uso de la Biodiversidad, México, 67 pp.

CONANP (Comisión Natural de Áreas Naturales Protegidas) (2007) Programa de Conservación y Manejo del Parque Nacional Lagunas de Montebello. Secretaría de Medio Ambiente y Recursos Naturales, México, D.F., 200 pp.

CONANP (Comisión Nacional de Áreas Naturales Protegidas) (2013) Monitoreo de aves en la Reserva de la Biosfera Ría Lagartos. Secretaría de Medio Ambiente y Recursos Naturales, México, 10 pp.

Durán, A. A. (2017) Datos preliminares sobre la influencia de la turbidez del agua y profundidad en el éxito de captura de presas por Megaceryle torquata (Aves, Alcedinidae). Revista Biodiversidad Neotropical, 7, 152-155.

http://dx.doi.org/10.18636/bioneotropical.v7i3.639

González-Espinosa, M., Ramírez-Marcial, N. (2013) Comunidades vegetales terrestres. Pp: 21-42. En: Comisión Nacional para el Conocimiento y Uso de la Biodiversidad (CONABIO) (Eds.) La biodiversidad en Chiapas: Estudio de Estado. México: CONABIO y Gobierno del Estado de Chiapas. México.

González-Ortega, M. A., Morales-Pérez, J. E. (1998) Distribución vertical de la avifauna en un bosque templado de Zinacatán, Chiapas, México. Acta Zoológica Mexicana (nueva serie), 76, 125-142. Disponible en: http://www.acuedi.org/doc/2041/distribuci\%26Atilde\%3B\%26sup3\%3Bn-verticalde-la-avifauna-en-un-bosque-templado-de-zinacantan-chiapas-m\%26Atilde\%3Bxico.html

Halffter, G., Moreno, C. (2005) Significado biológico de las diversidades alfa, beta y gamma. Pp: 5-18. En: G. Halffter, J. Soberón, P. Koleff, A. Melic. (Eds). Sobre diversidad biológica: El significado 
de las diversidades alfa, beta y gamma. España m3m: Monografías Tercer Milenio, Zaragoza, España.

Hill, M. O. (1973) Diversity and evenness: a unifying notation and its consequences. Ecology, 54, 427-432.

Hsieh, T. C., Ma, K. H., Chao, A. (2016) iNEXT: An R package for rarefaction and extrapolation of species diversity (H ill numbers). Methods in Ecology and Evolution, 7, 1451-1456.

Hutto, R., Pletschet, S., Hendricks, P. (1986) A fixed-radius point count method for nonbreeding and breeding season use. The Auk, 103, 593-602. Disponible en: //scholarworks.umt.edu/biosci_pubs/273

Jiménez-Cruz, R., Pulido-Arguello, H. (2009) Análisis para la obtención de la tasa de transformación en los ecosistemas del Parque Nacional Lagunas de Montebello del periodo 1998-2009. Comisión Nacional de Áreas Naturales Protegidas, Parque Nacional Lagunas de Montebello, México, 38 pp.

Jost, L. (2006) Entropy and diversity. Oikos, 113, 363-375. https://doi.org/10.1111/j.2006.0030-1299.14714.x

Kappelle, M., Brown, A. D. (2001) Bosques nublados del Neotrópico. INBIO, Instituto Nacional de Biodiversidad. Santo Domingo de Heredia, Costa Rica.

Lammertink, J. M., Rojas-Tomé, J. A., Casillas-Orona, F. M., Otto, R. L. (1996) Status and conservation of old-growth forests and endemic birds in the pine-oak zone of the Sierra Madre Occidental, Mexico. Verslagen Technische Gegevens, 69, 1-89.

Martínez-Sánchez, J. (2005) Frugívoros voladores y la dispersión de semillas en el Parque Nacional Lagunas de Montebello, Chiapas, México. El Colegio de la Frontera Sur, Chiapas, México, 39 pp.

Maya-García, E. E. (2017) Estimación del estado trófico de 18 lagos del Parque Nacional "Lagunas de Montebello”, Chiapas, México. Universidad Nacional Autónoma de México, México, 32 pp.

Melo, C., Cervantes, J. (1986) Propuestas para el programa integral de manejo y desarrollo del Parque Nacional Lagunas de Montebello. Investigaciones Geográficas, 16, 9-31.

Myers, N., Mittermeier, R., Mittermeier, C., da Fonseca, G., Kent, J. (2000) Biodiversity hot spots for conservation priorities. Nature, 403, 853-858.

Navarro, A. G., Peterson, A. T. (2007) Myadestes occidentalis (clarín jilguergo) residencia permanente. Distribución potencial, escala 1:1000000. Catálogo de metadatos geográficos, Comisión Nacional para el Conocimiento y Uso de la Biodiversidad (CONABIO), Disponible en: http://www.conabio.gob.mx/informacion/metadata/gis (último acceso febrero de 2018).

Navarro-Sigüenza, A. G., Lira-Noriega, A., Arizmendi, M. C., Berlanga, H., Koleff, P., GarcíaMoreno, J., Peterson, A. T. (2011) Áreas de conservación para las aves: hacia la integración de criterios de priorización. Pp: 109-129. En: P. Koleff, T. Urquiza-Haas (Coords.). Planeación para la conservación de la biodiversidad terrestre en México: retos en un país megadiverso. Comisión Nacional para el Conocimiento y Uso de la Biodiversidad -Comisión Nacional de Áreas Naturales Protegidas, México.

Navarro-Sigüenza, A. G., Rebón-Gallardo, F., Gordillo-Martínez, A., Towsend-Peterson, A., Berlanga-García, H., Sánchez-González, L. A. (2014) Biodiversidad de aves en México. Revista Mexicana de Biodiversidad, 85, 476-495. https://doi.org/10.7550/mb.41882

Paz, F., Marín, M. I., Medrano, E. R., Ibarra, F., Pascual, F. (2010) Elaboración de mapas multitemporales de bosque, a partir de imágenes LANDSAT, TM y ETM + y análisis de la degradación forestal y deforestación en Chiapas. Informe final consultoría para Conservación Internacional México A.C.

Pineda Diez de Bonilla, E., León-Cortés, J. L., Rangel-Salazar, J. L. (2012) Diversity of bird feeding guilds in relation to habitat heterogeneity and land-use cover in a human-modified landscape in southern Mexico. Journal of Tropical Ecology, 28, 369-376.

https://doi.org/10.1017/S026646741200034X 
Ralph, C. J., Geupel, G. R., Pyle, P., Martin, T. E., DeSante, D. F., Milá, B. (1996) Manual de métodos de campo para el monitoreo de aves terrestres. General Technical Report. Pacific Southwest Research Station-Department of Agriculture, California, $46 \mathrm{pp}$.

Ramírez-Albores, J. E. (2006) Variación en la composición de comunidades de aves en la Reserva de la Biosfera Montes Azules y áreas adyacentes, Chiapas, México. Biota Neotropical, 6, 1-19. Disponible en: http://www.biotaneotropica.org.br/v6n2/pt/abstract?article+bn03106022006

Rangel-Salazar, J. L., Enríquez, P. L. (2013) Estudio ecológico a largo plazo en la comunidad de aves en la Reserva Ecológica Cerro Huitepec, San Cristóbal de Las Casas, Chiapas. Pp: 342-344. En: CONABIO (Eds.). La Biodiversidad en Chiapas: Estudio de Estado. Comisión Nacional para el Conocimiento y Uso de la Biodiversidad (CONABIO) y Gobierno del Estado de Chiapas, México.

Rangel-Salazar, J. L., Enríquez, P. L., Sántiz-López, E. C. (2009) Variación de la diversidad de aves de sotobosque en el Parque Nacional Lagos de Montebello, Chiapas, México, Acta Zoológica Mexicana (nueva serie), 25, 479-495.

Rangel-Salazar, J. L., Enríquez-Rocha, P., Altamirano-González-Ortega, M. A., Macías-Caballero, C., Castillejos-Castellanos, E., González-Domínguez, P., Martínez-Ortega, J. A., VidalRodríguez, R. M. (2013) Diversidad de aves: un análisis espacial. Pp: 329-337. En: CONABIO (Eds.). La biodiversidad en Chiapas: Estudio de Estado. Comisión Nacional para el Conocimiento y Uso de la Biodiversidad (CONABIO) y Gobierno del Estado de Chiapas, México. https://doi.org/10.13140/2.1.2834.6888

Renjifo, L. M., Servat, G. P., Goerck, J. M., Loiselle, B. A., Blake, J. G. (1997) Patterns of species composition and endemism in the Northern Neotropics: a case for conservation of montane avifaunas. Ornithological Monographs, 48, 577-594.

Ruiz Bruce Taylor, M. D. M., Rangel Salazar, J. L., Enríquez, P. L., León-Cortés, J. L., GarcíaEstrada, C. (2017) Variation in hierarchical guild structure between two bird assemblages of a wetland in the Mexican Pacific. Digital Appendix 2. Revista de Biología Tropical, 65, 1540-1553. https://doi.org/10.15517/rbt.v65i4.26266

Sánchez-García, I. (2012) Dieta herpetófaga en una garcilla bueyera (Bubulcus ibis). Boletín de la Asociación Herpetológica Española, 23, 10-12.

Şekercioḡlu, C. H. (2006) Increasing awareness of avian ecological function. Trends in Ecology and Evolution, 21, 464-471. https://doi.org/10.1016/j.tree.2006.05.007

Şekercioğlu, C. H. (2012) Bird functional diversity and ecosystem services in tropical forests, agroforests and agricultural areas. Journal of Ornithology, 153, 153-161. https://doi.org/10.1007/s10336-012-0869-4

Şekercioḡlu, C. H., Ehrlich, P. R., Daily, G. C., Aygen, D., Goehring, D., Sandí, R. F. (2002) Disappearance of insectivorous birds from tropical fragments. Proceedings of National Academy of Science of the United States of America PNAS, 99, 263-267. https://doi.org/10.1073/pnas.012616199

SEMARNAT (Secretaría de Medio Ambiente y Recursos Naturales) (2010) Ecosistemas Terrestres, Disponible en: http://apps1.semarnat.gob.mx/dgeia/informe_12/pdf/Cap2_ecosistemas.pdf (último acceso abril de 2018).

Sobrino-Galdámez, I. (2011) Diversidad de aves en el área de manejo forestal de Coapilla, Chiapas. El Colegio de la Frontera Sur, México, 63 pp.

Sohdi, N. S, Şekercioḡlu, C. H., Barlow, J., Robinson, S. K. (2011) Conservation of tropical birds. WileyBlackwell, New Jersey, USA, 287 pp. https://doi.org/10.1002/978144434261

Stattersfield, A. J., Crosboy, M. J., Long, A. J., Wege, D. C. (1998) Endemic bird areas of the world. Priorities for Biodiversity Conservation. Birdlife Conservation Serie 7. Bird Life International, Cambridge, UK. 
Stotz, D., Fitzpatrick, J., Parker III, T., Moskovits, D. (1996) Ecology and conservation. The University of Chicago Press, Chicago, USA, 478 pp.

Suárez-García, O., González-García, F., Celis-Murillo, A. (2017) Entendiendo la complementariedad de dos métodos de muestreo en el estudio de comunidades de aves de un bosque mesófilo de montaña en temporada reproductiva. Revista Mexicana de Biodiversidad, 88, 880-887. https://doi.org/10.1016/j.rmb2017.10.029

The CornellLab of Ornithology (2017) Cornell University, NY. Disponible en: www.birds.cornell.edu (último acceso septiembre de 2017).

Toledo, V. M. (1994) La diversidad biológica de México, nuevos retos para la investigación en los noventa. Ciencias, 34, 43-57.

Van Perlo, B. (2006) A field guide to Birds of México and Central America. Princeton University Press, USA, $336 \mathrm{pp}$. 
Apéndice 1. Clasificación en gremios tróficos de la comunidad de aves del Parque Nacional Lagunas de Montebello, Chiapas, México. Estrato de forrajeo (Es): agua ( $\mathrm{Ag}$ ), terrestre (Te), sotobosque (So), estrato medio (Em), estrato alto (Ea) y aéreo (V). Sensibilidad a la perturbación (SP): baja (B), media (M) y alta (A). Estacionalidad (St): Residente

(R) y migratoria latitudinal (MLD). Categoría de riesgo (NOM 059-2010) (CR): Protección especial (Pr), amenazada (A) y peligro de extinción (P). Tipo de cobertura donde fueron avistados: bosque de pino (BP), bosque de pino-encino (BPE), bosque de pino-encino-liquidambar (BPEL), bosque mesófilo de montaña (BMM), vegetación riparia (VR) y zonas con intervención (ZI). El listado de especies fue con base al AOU (2017).

\begin{tabular}{|c|c|c|c|c|c|}
\hline $\begin{array}{l}\text { Gremio trófico } \\
\text { Especies }\end{array}$ & Es & SP & $\mathbf{S t}$ & $\mathbf{C R}$ & Tipo de vegetación \\
\hline \multicolumn{6}{|c|}{ (FIVp) Frugívoro-Insectívoro-Vertebrados pequeños } \\
\hline Aulacorhynchus prasinus & $\mathrm{Ea}$ & M & $\mathrm{R}$ & A & BPEL, BMM, ZI \\
\hline Catharus frantzii & Te, So & M & $\mathrm{R}$ & A & BPE, BPEL, ZI \\
\hline Penelopina nigra & $\mathrm{Ea}$ & A & $\mathrm{R}$ & $\mathrm{P}$ & BP, BPE, BPEL, BMM, ZI \\
\hline Piaya cayana & $\mathrm{Ea}$ & $\mathrm{B}$ & $\mathrm{R}$ & & BPE, BMM \\
\hline \multicolumn{6}{|c|}{ (G) Granívoro } \\
\hline Sporophila funerea & So, Em & $\mathrm{B}$ & $\mathrm{R}$ & & ZI \\
\hline Spinus notatus & $\mathrm{Ea}$ & M & $\mathrm{R}$ & & $\mathrm{BP}, \mathrm{ZI}$ \\
\hline Spinus psaltria & $\mathrm{Ea}$ & $\mathrm{B}$ & $\mathrm{R}$ & & VR \\
\hline Volatinia jacarina & Te, So & B & $\mathrm{R}$ & & ZI \\
\hline Zonotrichia capensis & Te, So & $\mathrm{B}$ & $\mathrm{R}$ & & $\mathrm{BP}, \mathrm{ZI}$ \\
\hline
\end{tabular}

Zonotrichia capensis

\section{(GFI) Granívoro-Frugívoro-Insectívoro}

\begin{tabular}{|c|c|c|c|c|c|}
\hline Agelaius phoeniceus & $\mathrm{Te}, \mathrm{Em}$ & B & $\mathrm{R}$ & & BP, BPE, BPEL, VR, ZI \\
\hline Aimophila rufescens & $\mathrm{Te}$ & B & $\mathrm{R}$ & & $\mathrm{BP}$ \\
\hline Aspatha gularis & So & A & $\mathrm{R}$ & $\mathrm{P}$ & BP, BPEL, BMM, ZI \\
\hline Atlapetes albinucha & Te, So & M & $\mathrm{R}$ & & BPEL \\
\hline Basileuterus culicivorus & So, Em & M & $\mathrm{R}$ & & BPE, BPEL, BMM, ZI \\
\hline Basileuterus rufifrons & So & B & $\mathrm{R}$ & & $\mathrm{BP}, \mathrm{ZI}$ \\
\hline Campephilus guatemalensis & $\mathrm{Ea}$ & M & $\mathrm{R}$ & $\operatorname{Pr}$ & ZI \\
\hline Catharus aurantiirostris & Te, So & B & $\mathrm{R}$ & & $\mathrm{BP}, \mathrm{ZI}$ \\
\hline Catharus mexicanus & Te, So & M & $\mathrm{R}$ & $\operatorname{Pr}$ & BP, BPE, BPEL, BMM, VR, ZI \\
\hline Catharus ustulatus & Te, So & M & MLD & & ZI \\
\hline Chlorospingus flavopectus & So, Em & M & $\mathrm{R}$ & & BP, BPE, BPEL, BMM, VR, ZI \\
\hline Coccyzus americanus & $\mathrm{Em}, \mathrm{Ea}$ & $\mathrm{M}$ & MLD & & $\mathrm{BPE}$ \\
\hline Contopus sordidulus & $\mathrm{Ea}$ & M & $\mathrm{R}$ & & BP, BPEL, ZI \\
\hline Crypturellus cinnamomeus & $\mathrm{Te}$ & B & $\mathrm{R}$ & $\operatorname{Pr}$ & BP, BPE, BPEL, ZI \\
\hline Cyanolyca pumilo & $\mathrm{Ea}$ & A & $\mathrm{R}$ & A & ZI \\
\hline Cyclarhis gujanensis & Em, Ea & B & $\mathrm{R}$ & & BP, BPE, BMM, VR, ZI \\
\hline Dives dives & Te, Em & B & $\mathrm{R}$ & & BP, BPE, BPEL, BMM, ZI \\
\hline Dryocopus lineatus & $\mathrm{Ea}$ & B & $\mathrm{R}$ & & BPEL, VR \\
\hline Dumetella carolinensis & $\mathrm{Te}$ & B & MLD & & BP, BPE, BPEL, BMM, VR, ZI \\
\hline Euphonia hirundinacea & $\mathrm{Ea}$ & $\mathrm{B}$ & $\mathrm{R}$ & & $\mathrm{ZI}$ \\
\hline Geothlypis formosa & So, Em & M & MLD & & $\mathrm{BP}, \mathrm{ZI}$ \\
\hline Hylocichla mustelina & So & M & MLD & & BP, BPE, BPEL, BMM, VR, ZI \\
\hline Lepidocolaptes affinis & Em & M & $\mathrm{R}$ & & BP, BPE, BPEL, BMM \\
\hline Lepidocolaptes souleyetii & So, Em & B & $\mathrm{R}$ & & $\mathrm{BP}, \mathrm{BPEL}$ \\
\hline Leptotila verreauxi & Te, So & B & $\mathrm{R}$ & $\operatorname{Pr}$ & BP, BPE, BPEL, ZI \\
\hline Melanotis hypoleucus & So & M & $\mathrm{R}$ & & ZI \\
\hline Mionectes oleagineus & So, Ea & M & $\mathrm{R}$ & & BPEL, BMM \\
\hline Myadestes occidentalis & $\mathrm{Ea}$ & M & $\mathrm{R}$ & $\operatorname{Pr}$ & BP, BPE, BPEL, BMM, VR, ZI \\
\hline Myadestes unicolor & $\mathrm{Em}, \mathrm{Ea}$ & M & $\mathrm{R}$ & A & BP, BPE, BMM, ZI \\
\hline
\end{tabular}




\begin{tabular}{|c|c|c|c|c|c|}
\hline $\begin{array}{l}\text { Gremio trófico } \\
\text { Especies }\end{array}$ & Es & SP & St & $\mathbf{C R}$ & Tipo de vegetación \\
\hline Myiarchus tuberculifer & Em, Ea & $\mathrm{B}$ & $\mathrm{R}$ & & BP, BPEL, VR, ZI \\
\hline Myiodynastes luteiventris & Em, Ea & $\mathrm{B}$ & MLD & & $\mathrm{BP}, \mathrm{ZI}$ \\
\hline Myiozetetes similis & $\mathrm{Em}, \mathrm{Ea}$ & $\mathrm{B}$ & $\mathrm{R}$ & & $\mathrm{ZI}$ \\
\hline Oncostoma cinereigulare & So, Em & $\mathrm{B}$ & $\mathrm{R}$ & & BP, BPE, BPEL, BMM, ZI \\
\hline Ortalis vetula & $\mathrm{Te}, \mathrm{Ea}$ & $\mathrm{B}$ & $\mathrm{R}$ & & BP, BPE, BPEL, BMM, VR, ZI \\
\hline Patagioenas fasciata & $\mathrm{Ea}$ & M & $\mathrm{R}$ & $\operatorname{Pr}$ & $\mathrm{BP}, \mathrm{BPE}, \mathrm{BPEL}, \mathrm{BMM}$ \\
\hline Patagioenas flavirostris & $\mathrm{Ea}$ & $\mathrm{M}$ & $\mathrm{R}$ & & ZI \\
\hline Pharomachrus mocinno & Em & M & $\mathrm{R}$ & $\mathrm{P}$ & BPEL, BMM \\
\hline Piranga flava & $\mathrm{Ea}$ & $\mathrm{B}$ & $\mathrm{R}$ & & BP, BPE, ZI \\
\hline Pionus senilis & $\mathrm{Ea}$ & M & $\mathrm{R}$ & A & BPEL, BMM \\
\hline Setophaga pensylvanica & $\mathrm{Em}, \mathrm{Ea}$ & $\mathrm{B}$ & MLD & & BPE, BPEL \\
\hline Setophaga petechia & $\mathrm{Ea}$ & $\mathrm{B}$ & $\mathrm{R}$ & & BPEL \\
\hline Setophaga virens & $\mathrm{Em}, \mathrm{Ea}$ & M & MLD & & BP, BPE, BPEL, BMM, ZI \\
\hline Sporophila torqueola & So & $\mathrm{B}$ & $\mathrm{R}$ & & $\mathrm{ZI}$ \\
\hline Sturnella magna & $\mathrm{Te}$ & $\mathrm{B}$ & $\mathrm{R}$ & & VR \\
\hline Stilpnia larvata & $\mathrm{Ea}$ & $\mathrm{B}$ & $\mathrm{R}$ & & ZI \\
\hline Thraupis Abbas & $\mathrm{Ea}$ & $\mathrm{B}$ & $\mathrm{R}$ & & $\mathrm{ZI}$ \\
\hline Troglodytes aedon & Te, So & $\mathrm{B}$ & $\mathrm{R}$ & & BP, BPE, BPEL, BMM, VR, ZI \\
\hline Trogon collaris & $\mathrm{Em}, \mathrm{Ea}$ & M & $\mathrm{R}$ & $\operatorname{Pr}$ & BP, BPE, BPEL, BMM, ZI \\
\hline Trogon mexicanus & $\mathrm{Em}, \mathrm{Ea}$ & M & $\mathrm{R}$ & & $\mathrm{BP}, \mathrm{BPE}, \mathrm{BPEL}, \mathrm{VR}, \mathrm{ZI}$ \\
\hline Turdus assimilis & So, Em & M & $\mathrm{R}$ & & $\mathrm{ZI}$ \\
\hline Turdus infuscatus & $\mathrm{Em}, \mathrm{Ea}$ & A & $\mathrm{R}$ & $\mathrm{P}$ & BP, BPE, BPEL, BMM, ZI \\
\hline Tyrannus melancholicus & $\mathrm{Ea}$ & $\mathrm{B}$ & $\mathrm{R}$ & & $\mathrm{ZI}$ \\
\hline Zenaida asiática & $\mathrm{Te}, \mathrm{Em}$ & $\mathrm{B}$ & $\mathrm{R}$ & & BP, BPE, BPEL, BMM, VR, ZI \\
\hline \multicolumn{6}{|c|}{ I (Insectívoro) } \\
\hline Atlapetes albinucha (gutturalis) & Te, So & $\mathrm{M}$ & $\mathrm{R}$ & & $\mathrm{BP}, \mathrm{VR}, \mathrm{ZI}$ \\
\hline Clibanornis rubiginosus & So & M & $\mathrm{R}$ & A & BMM, ZI \\
\hline Campylorhynchus zonatus & $\mathrm{Em}, \mathrm{Ea}$ & $\mathrm{B}$ & $\mathrm{R}$ & & $\mathrm{BP}, \mathrm{BPE}, \mathrm{BPEL}, \mathrm{VR}, \mathrm{ZI}$ \\
\hline Cantorchilus modestus & So & $\mathrm{B}$ & $\mathrm{R}$ & & $\mathrm{BP}, \mathrm{BMM}, \mathrm{VR}, \mathrm{ZI}$ \\
\hline Colaptes auratus & $\mathrm{Te}, \mathrm{Ea}$ & $\mathrm{B}$ & $\mathrm{R}$ & & BP, BPE, BPEL, BMM, VR, ZI \\
\hline Colaptes rubiginosus & $\mathrm{Ea}$ & $\mathrm{B}$ & $\mathrm{R}$ & & BP, BPE, BPEL, BMM, VR, ZI \\
\hline Contopus cooperi & $\mathrm{Em}, \mathrm{Ea}$ & NA & MLD & & $\mathrm{BP}, \mathrm{VR}, \mathrm{ZI}$ \\
\hline Contopus pertinax & $\mathrm{Ea}$ & M & $\mathrm{R}$ & & $\mathrm{BP}$ \\
\hline Elaenia flavogaster & $\mathrm{Ea}$ & $\mathrm{B}$ & $\mathrm{R}$ & & $\mathrm{ZI}$ \\
\hline Empidonax affinis & $\mathrm{Em}, \mathrm{Ea}$ & M & $\mathrm{R}$ & & BP, BPE, BPEL, BMM, VR, ZI \\
\hline Geothlypis trichas & So & $\mathrm{B}$ & MLD & & BPEL, ZI \\
\hline Henicorhina leucophrys & So & M & $\mathrm{R}$ & & BPE, BPEL, ZI \\
\hline Myioborus miniatus & $\mathrm{Em}, \mathrm{Ea}$ & $\mathrm{B}$ & $\mathrm{R}$ & & BP, BPE, BPEL, BMM, VR, ZI \\
\hline Myioborus pictus & So, Em & $\mathrm{M}$ & $\mathrm{R}$ & & $\mathrm{ZI}$ \\
\hline Pheugopedius maculipectus & So, Em & $\mathrm{B}$ & $\mathrm{R}$ & & BP, BPE, BPEL, BMM, ZI \\
\hline Dryobate villosus & $\mathrm{Em}, \mathrm{Ea}$ & M & $\mathrm{R}$ & & $\mathrm{BP}, \mathrm{BPEL}$ \\
\hline Piranga bidentata & $\mathrm{Ea}$ & M & $\mathrm{R}$ & & BP, BPEL, ZI \\
\hline Setophaga chrysoparia & So & M & MLD & $\mathrm{P}$ & BMM \\
\hline Setophaga fusca & $\mathrm{Em}, \mathrm{Ea}$ & M & MLD & & BP, BPE, BPEL, BMM \\
\hline Setophaga graciae & $\mathrm{Ea}$ & M & MLD & & BP, BPE, BPEL, ZI \\
\hline Setophaga townsendi & Em, Ea & M & MLD & & BP, BPE, BPEL, VR, ZI \\
\hline Sialia sialis & Em, Ea & $\mathrm{B}$ & $\mathrm{R}$ & & $\mathrm{BP}, \mathrm{BPEL}, \mathrm{VR}, \mathrm{ZI}$ \\
\hline
\end{tabular}




\begin{tabular}{|c|c|c|c|c|c|}
\hline $\begin{array}{l}\text { Gremio trófico } \\
\text { Especies }\end{array}$ & Es & SP & St & CR & Tipo de vegetación \\
\hline Xiphorhynchus erythropygius & Em & M & $\mathrm{R}$ & A & BP, BPE, BPEL, BMM \\
\hline Xiphorhynchus flavigaster & So, Em & $\mathrm{M}$ & $\mathrm{R}$ & & $\mathrm{BP}, \mathrm{BPE}$ \\
\hline \multicolumn{6}{|c|}{ (IAcVp) Insectívoro-Invertebrados acuáticos-Vertebrados acuáticos } \\
\hline Buteogallus anthracinus & $\mathrm{Ea}$ & $\mathrm{M}$ & $\mathrm{R}$ & $\operatorname{Pr}$ & BPEL, ZI \\
\hline Dendrocygna autumnalis & $\mathrm{Te}, \mathrm{Ag}$ & $\mathrm{B}$ & $\mathrm{R}$ & & VR \\
\hline Megaceryle torquata & So, Em & $\mathrm{B}$ & $\mathrm{R}$ & & $\mathrm{BP}$ \\
\hline Nycticorax nycticorax & $\mathrm{Te}, \mathrm{Ag}$ & $\mathrm{B}$ & $\mathrm{R}$ & & $\mathrm{BP}, \mathrm{VR}$ \\
\hline \multicolumn{6}{|c|}{ (IF) Insectívoro-Frugívoro } \\
\hline Mniotilta varia & So & $\mathrm{B}$ & MLD & & BP, BPE, BPEL, ZI \\
\hline Piranga rubra & $\mathrm{Ea}$ & $\mathrm{B}$ & MLD & & $\mathrm{ZI}$ \\
\hline \multicolumn{6}{|c|}{ (IN) Insectívoro-Nectarívoro } \\
\hline Amazilia cyanocephala & $\mathrm{Em}, \mathrm{Ea}$ & $\mathrm{M}$ & $\mathrm{R}$ & & BP, BPE, BPEL, BMM, VR, ZI \\
\hline Colibri thalassinus & $\mathrm{Em}, \mathrm{Ea}$ & $\mathrm{B}$ & $\mathrm{R}$ & & $\mathrm{BP}, \mathrm{BPE}, \mathrm{BPEL}, \mathrm{BMM}, \mathrm{VR}, \mathrm{ZI}$ \\
\hline Cyanerpes cyaneus & $\mathrm{Ea}$ & $\mathrm{B}$ & $\mathrm{R}$ & & $\mathrm{ZI}$ \\
\hline Eugenes fulgens & So, Em & M & $\mathrm{R}$ & & $\mathrm{BMM}$ \\
\hline Hylocharis leucotis & So, Em & M & $\mathrm{R}$ & & BP, BPEL, BMM, ZI \\
\hline Lampornis viridipallens & So, Em & M & $\mathrm{R}$ & $\operatorname{Pr}$ & $\mathrm{BP}, \mathrm{BPE}, \mathrm{BPEL}, \mathrm{BMM}$ \\
\hline Tilmatura dupontii & So, Em & $\mathrm{M}$ & $\mathrm{R}$ & $\mathrm{A}$ & $\mathrm{BP}, \mathrm{BMM}, \mathrm{VR}$ \\
\hline \multicolumn{6}{|c|}{ (Ivp) Insectívoro-Vertebrados pequeños } \\
\hline Accipiter striatus (chionogaster) & $\mathrm{Ea}$ & $\mathrm{M}$ & $\mathrm{R}$ & $\operatorname{Pr}$ & BPE, BPEL \\
\hline Basileuterus belli & So & M & $\mathrm{R}$ & & $\mathrm{BMM}$ \\
\hline Bubulcus ibis & $\mathrm{Te}, \mathrm{Ag}$ & $\mathrm{B}$ & $\mathrm{R}$ & & $\mathrm{ZI}$ \\
\hline Rupornis magnirostris & $\mathrm{Ea}$ & $\mathrm{B}$ & $\mathrm{R}$ & & $\mathrm{BP}, \mathrm{BPEL}, \mathrm{VR}$ \\
\hline Buteo platypterus & $\mathrm{Ea}$ & $\mathrm{M}$ & MLD & $\operatorname{Pr}$ & $\mathrm{BP}, \mathrm{VR}$ \\
\hline Dendrocolaptes picumnus & $\mathrm{Em}$ & A & $\mathrm{R}$ & A & $\mathrm{BP}$ \\
\hline Elanus leucurus & $\mathrm{Ea}, \mathrm{V}$ & $\mathrm{B}$ & $\mathrm{R}$ & & VR \\
\hline Falco rufigularis & $\mathrm{Ea}, \mathrm{V}$ & $\mathrm{B}$ & $\mathrm{R}$ & & BMM, ZI \\
\hline Pandion haliaetus & $\mathrm{Ag}$ & $\mathrm{M}$ & MLD & & BPE, BPEL \\
\hline \multicolumn{6}{|c|}{ (O) Omnívoro } \\
\hline Aphelocoma unicolor & $\mathrm{Ea}$ & $\mathrm{M}$ & $\mathrm{R}$ & $\mathrm{A}$ & BP, BPE, BPEL, BMM, VR, ZI \\
\hline Cardellina pusilla & So & $\mathrm{B}$ & MLD & & $\mathrm{BP}, \mathrm{BPE}, \mathrm{BPEL}, \mathrm{ZI}$ \\
\hline Cathartes aura & $\mathrm{Te}, \mathrm{Ag}$ & $\mathrm{B}$ & $\mathrm{R}$ & & $\mathrm{BP}, \mathrm{VR}$ \\
\hline Cyanolyca cucullata & So, Em & M & $\mathrm{R}$ & $\mathrm{A}$ & BPE, BMM \\
\hline Icterus chrysater & $\mathrm{Ea}$ & $\mathrm{B}$ & $\mathrm{R}$ & & BP, BPE, BPEL, BMM, VR, ZI \\
\hline Melanerpes aurifrons & $\mathrm{Em}, \mathrm{Ea}$ & $\mathrm{B}$ & $\mathrm{R}$ & & ZI \\
\hline Mimus gilvus & $\mathrm{Ea}$ & $\mathrm{B}$ & $\mathrm{R}$ & & BPEL, ZI \\
\hline Psilorhinus morio & $\mathrm{Ea}$ & $\mathrm{B}$ & $\mathrm{R}$ & & BP, BPE, BPEL, BMM, VR, ZI \\
\hline Quiscalus mexicanus & $\mathrm{Te}, \mathrm{Ea}$ & $\mathrm{B}$ & $\mathrm{R}$ & & BP, BPE, BPEL, ZI \\
\hline Ramphocelus sanguinolentus & So, Em & $\mathrm{B}$ & $\mathrm{R}$ & & ZI \\
\hline Saltator atriceps & So, Em & M & $\mathrm{R}$ & & ZI \\
\hline Saltator maximus & $\mathrm{Em}, \mathrm{Ea}$ & $\mathrm{B}$ & $\mathrm{R}$ & & ZI \\
\hline Setophaga coronata & So, Em & $\mathrm{B}$ & MLD & A & $\mathrm{ZI}$ \\
\hline Thamnophilus doliatus & So, Em & B & $\mathrm{R}$ & & BP, BPE, BMM, VR, ZI \\
\hline Turdus grayi & $\mathrm{Te}, \mathrm{Em}$ & $\mathrm{B}$ & $\mathrm{R}$ & & $\mathrm{BP}, \mathrm{BPE}, \mathrm{BPEL}, \mathrm{BMM}, \mathrm{VR}, \mathrm{ZI}$ \\
\hline
\end{tabular}

